\title{
Surgical site dressing practice
}

\author{
S. Sijimol ${ }^{\mathrm{a}}$, Leena Sequira ${ }^{\mathrm{b}, *}$, Prima Jenevive Jyothi D'Souza ${ }^{\mathrm{a}}$, Vandana Kalwaje Eshwara ${ }^{\mathrm{c}}$ \\ ${ }^{a}$ Manipal College of Nursing Manipal, Manipal Academy of Higher Education, Manipal, 576104, India \\ ${ }^{\mathrm{b}}$ Manipal School of Nursing Manipal, Manipal Academy of Higher Education, Manipal, 576104, India \\ ${ }^{\mathrm{c}}$ Department of Microbiology, Kasturba Medical College, Manipal Academy of Higher Education, Manipal, 576104, India
}

\section{A B S T R A C T}

Background: Surgical site infections (SSI) compose of $20 \%$ of all healthcare-associated infections.

Materials and methods: Cross-sectional survey was conducted to assess the surgical site dressing practices. Event sampling was adopted, and 88 dressing procedures were observed. The perceived barriers in following infection control measures were assessed by questionnaire.

Results: There was a lack of aseptic wound dressing practices among healthcare professionals. Heavy workload and inadequate staff to assist in dressing procedures were considered as the major barriers.

Conclusion: Evidence-Based practice guidelines should be established, and healthcare professionals should adhere to the infection control measures to reduce the incidence of SSI.

\section{Introduction}

Surgical site infections (SSI) are the most common postoperative complication occurring in surgical patients. SSI causes substantial postoperative morbidity and mortality; it extends the hospital stay and increases the health care cost. According to "The Burden of Health CareAssociated Infection Worldwide, WHO," in developing countries, SSI is the most frequent type of infection. The literature reports in the US the incidence of SSI is $20 \%$, which is next after the urinary tract infection of $36 \%{ }^{1}$ Incidence of SSI in general surgeries at Brazilian hospital was $3.4 \% .^{2}$ A study done in India, among 300 surgical wounds from General Surgery and Obstetrics and Gynecology wards shows the prevalence of SSI is $6 \%$ in one year. ${ }^{3}$ The prevalence of SSI in general surgery wards was $12.5 \%$, among which superficial incision SSI was most common. ${ }^{4} \mathrm{~A}$ study conducted in a tertiary care hospital reports a prevalence rate of $4.3 \%$, superficial surgical site infection, and most of the health professionals did not practice all the steps of the wound dressing. ${ }^{5}$

Poor practice with regard to wearing gloves and disposal of wound dressing material was observed. ${ }^{6}$ Appropriate dressing practices were not followed by many of the health professionals. ${ }^{7}$ Healthcare professionals, including the individual doctors and nurses, should be aware of the surgical site infections and the burden it carries both on the patient and hospital. Steps should be taken to control and prevent surgical site infections. Identifying the surgical site infections helps the healthcare professionals in carrying out the prompt action at the earliest in the prevention of the infection. Improper handling of the surgical wound, non-aseptic dressing procedures always contributes to surgical site infections. Reviewing, revising, and adherence to the protocol are essential in preventing and reducing the burden of surgical site infections. Healthcare professionals may have certain barriers which hinders in practicing the appropriate techniques while dressing the surgical wound. Identification of these barriers and taking prompt action would help in reducing the risk of developing SSI. ${ }^{1}$

Hence the present study was conducted in a tertiary care hospital with the objective to assess the surgical site dressing practices and to explore the perceived barriers to follow infection control measures in the dressing practices among healthcare professionals.

\section{Materials and methods}

The study was conducted at a tertiary care teaching hospital, and study design was a cross sectional survey. Healthcare professionals were observed for the practice of surgical site dressing. The sample size is calculated based on the estimation for proportion for the events of postoperative surgical wound dressing practice. An event sampling technique was adopted to assess the surgical site dressing practice. Tools used for data collection were demographic proforma, observation checklist on the postoperative surgical site dressing practice, and questionnaire on perceived barriers to follow infection control measures in surgical wound dressing practice. The tool demographic proforma consisted of age, gender, profession, educational qualification, years of experience, and any course, seminar, or workshop attended

\footnotetext{
* Corresponding author.

E-mail addresses: Sijimw15@gmail.com (S. Sijimol), leena.sequ@manipal.edu (L. Sequira), primajj@gmail.com (P.J.J. D'Souza), Vandana.ke@manipal.edu (V.K. Eshwara).
} 
Table 1

Frequency (f) and Percentage (\%) of the participant Characteristics.

\begin{tabular}{lll}
\hline $\mathrm{N}=38$ & & \\
\hline Variables & Frequency & Percentage \\
\cline { 2 - 3 } & $\mathrm{f}$ & $\%$ \\
\hline Age & & \\
20-25 years & 21 & 55.3 \\
26-30 years & 17 & 44.7 \\
Gender & & \\
Male & 20 & 52.6 \\
$\quad$ Female & 18 & 47.4 \\
Profession & & \\
$\quad$ Doctor & 38 & 100 \\
Educational qualification & & \\
$\quad$ MBBS & 24 & 63.2 \\
$\quad$ Post-graduation in surgery & 14 & 36.8 \\
Any course, seminar or workshop attended related to surgical site dressing or SSI \\
$\quad$ Yes & 23 & 60.5 \\
$\quad$ No & 15 & 39.5 \\
\hline
\end{tabular}

concerning surgical site dressing or surgical site infection. The practice of surgical site dressing was observed with the check list. The checklist was validated by seven experts, and the reliability was established by interrater reliability test $(r=0.95)$. The checklist consisted of 17 items, which include the procedural steps of performing the surgical site dressing, with two options as 'performed' and 'not performed.' Each item was analysed in frequency and percentage as performed and not performed. The third tool, a self-administered questionnaire on barriers to follow infection control measures, was used, which consisted of 12 items with the response of 'yes' or 'no.' The barrier tool was validated by seven experts, and the reliability was established by test-retest reliability $(r=0.95)$. Each item was analysed in frequency and percentage. After obtaining the ethical permissions, (IEC- 771/2016, CTRI Ref/2016/12/012905 (B)), concealed observation of 88 events of postoperative surgical site dressing was done in the general surgery units. The data was collected for a period of four weeks. The inclusion criteria were the wound dressing practices performed on patients who had undergone open abdominal surgery without any pre-existing surgical site infection. At one time, the researcher observed only one event of surgical site dressing practice, and each participant was observed not more than five times. Following which the demographic proforma and questionnaire on barriers to follow infection control measures were administered. Informed consent was obtained from the participants, and confidentiality was maintained during the data collection process and throughout the study.

The sample size was calculated based on the estimation for proportion for the events of postoperative surgical wound dressing practice.

$\mathrm{N}=\frac{\mathrm{Z}_{1-\alpha / 2}^{2} \mathrm{p} \cdot \mathrm{q}}{\mathrm{d}^{2}}$

Where.

$$
\begin{aligned}
& \mathrm{n}=\text { minimum sample size required } \\
& \mathrm{Z}_{1-\alpha / 2}^{2}=\text { confidence level at } 95 \%(1.96) \\
& \mathrm{p}=\text { estimated proportion (Based on the pilot study findings, } \\
& \mathrm{p}=0.65 \text { ) } \\
& \mathrm{q}=1-\mathrm{p} \\
& \mathrm{d}=\text { clinical significant difference, relative precision at } 10 \%
\end{aligned}
$$

The sample size for the study was estimated to be 87 .

Collected data were entered in Statistical Package for Social Sciences (SPSS 16 version). Descriptive statistics (frequency and percentage) was used to describe the study findings. ${ }^{8,9}$

\section{Results}

The participant characteristics showed that majority (55.3\%) of the healthcare professionals were between the age group of 20-25 years, $52.6 \%$ were male doctors, $63.2 \%$ with the educational qualification of MBBS, and majority (60.5\%) had attended course, seminar or workshop concerning surgical site dressing [Table 1].

The findings from the observation of practice reveal that majority of the dressings $(68.2 \%)$ were done immediately by not exposing the wound for a longer period. The majority $(84.1 \%)$ of healthcare professionals wore sterile gloves without contamination, $75 \%$ had not practiced hand hygiene after opening the wound dressing, and most (53.4\%) had not practiced hand hygiene after the procedure [Table 2].

The study results showed that the major barriers for not practicing the appropriate technique of wound dressing were, time-consuming procedure, heavy workload, lack of adequate staff to assist in dressing procedure and lack of sufficient supplies [Table 3].

\section{Discussion}

Findings of the present study report that $75 \%$ of the participants had not practiced hand hygiene after opening the wound and 53.4\% had not practiced hand hygiene after the procedure. The present study findings were supported by the following studies. A cross sectional observational

Table 2

Frequency (f) and percentage (\%) of the postoperative surgical wound dressing practice.

$\mathrm{N}=88$
Wound dressing practice

Decontaminates the hands using the correct technique, with liquid soap and water or an alcohol hand rub is used Dries the hands before wearing gloves.

The dressing is done immediately by not exposing the wound to the external environment for a longer period.

Wears clean, disposable gloves to remove the old dressing.

Performs hand hygiene after opening the wound

Uses PPE like mask, goggles, and apron as needed.

Wears sterile gloves without contaminating them.

Uses sterile technique for opening the dressing pack.

Receives articles and solutions provided by another person without contaminating the sterile field.

Drapes the wound site with a sterile sheet or hole towel.

Uses sterile cotton or gauze to clean the wound.

Cleans the wound with strokes from "top to bottom" or "centre to the periphery"

Uses sterile cotton or gauze to cleanse wound from "clean to dirty"

Uses new gauze for each wipe.

Follows sterile technique throughout the procedure

Performs hand washing after the procedure.

Disposes the waste into the respective waste bin as per institutional policy.

\begin{tabular}{llll}
$\begin{array}{l}\text { Performed } \\
\text { (f) }\end{array}$ & $\%$ & \multicolumn{2}{c}{ Not performed } \\
69 & 78.4 & 19 & $\%$ \\
52 & 59.1 & 36 & 21.6 \\
60 & 68.2 & 28 & 40.9 \\
30 & 34.1 & 58 & 31.8 \\
22 & 25 & 66 & 65.9 \\
25 & 28.4 & 63 & 75 \\
74 & 84.1 & 14 & 71.6 \\
53 & 60.2 & 35 & 15.9 \\
60 & 68.2 & 28 & 39.8 \\
36 & 40.9 & 52 & 31.8 \\
80 & 90.9 & 8 & 59.1 \\
44 & 50 & 44 & 9.1 \\
57 & 64.8 & 31 & 50 \\
62 & 70.5 & 26 & 35.2 \\
26 & 29.5 & 62 & 29.5 \\
41 & 46.6 & 47 & 70.5 \\
73 & 83 & 15 & 53.4 \\
\end{tabular}


Table 3

Frequency (f) and Percentage (\%) of the Barriers to follow Infection Control Measures in the Surgical Wound Dressing Practice.

\section{$\mathrm{N}=38$}

\begin{tabular}{lllll}
\hline Perceived barriers & \multicolumn{2}{l}{ Yes } & \multicolumn{2}{l}{ No } \\
\cline { 2 - 6 } & (f) & $\%$ & (f) & $\%$ \\
\hline The surgical wound dressing is a time-consuming task & 23 & 60.5 & 15 & 39.5 \\
Laziness to follow the correct procedures & 2 & 5.3 & 36 & 94.7 \\
Hand washing station is not situated nearby. & 7 & 18.4 & 31 & 81.6 \\
I was not trained on the dressing protocol & 2 & 5.3 & 36 & 94.7 \\
I often do not remember all the procedure steps & 5 & 13.2 & 33 & 86.8 \\
Heavy workload & 24 & 63.2 & 14 & 36.8 \\
Lack of supervision by seniors & 6 & 15.8 & 32 & 84.2 \\
I perceive that there is minimal chance of acquiring & 14 & 36.8 & 24 & 63.2 \\
$\quad$ infection due to dressing procedure & & & & \\
Lack of sufficient supplies & 6 & 15.8 & 32 & 84.2 \\
Lack of adequate staff to assist in dressing procedure & 15 & 39.5 & 23 & 60.5 \\
Lack of knowledge of scientific rationale for the steps of & 6 & 15.8 & 32 & 84.2 \\
$\quad$ the procedure. & & & & \\
Inadequate cooperation from the patient & 15 & 39.5 & 23 & 60.5 \\
& & & & \\
\hline
\end{tabular}

study conducted to analyze the quality assessment in a wound dressing shows that from the 168 dressings observed, there was a lack of hand washing before and also after the procedure. ${ }^{10}$ An experimental study conducted to minimize the procedural inconsistency and increase compliance with infection control measures during a change of dressings revealed that $60 \%$ of the participants were not following the hand hygiene practice. ${ }^{11}$ A quantitative, observational study conducted to verify the use of the steps in the execution of dressings, concluded that only $16.6 \%$ of the professionals washed hand before performing the dressing and $56.6 \%$ of professionals put in sterile gloves. Only $30.9 \%$ of them disposed of soiled dressing in the disposable bag. ${ }^{12}$

In the present study, $60.5 \%$ of the health care professionals perceived surgical wound dressing is a time consuming task. $63.2 \%$ of the health care professionals perceived heavy workload as a barrier. $39.5 \%$ of the health care professionals perceived that there is a lack of adequate staff to assist in the dressing procedure.

The present study findings are supported by the following studies. An environmental scan to find the barriers of management of surgical wound conducted among nursing, product supply, and inventory control professionals showed that historical and traditional pretexts, economic constraints, clinical expertise and knowledge and factors relating to the patient were the major barriers. ${ }^{13}$ Systematic review done to assess the barriers to the implementation of best practice in wound care found that inappropriate training and support, instability of health services, lack of education and support, lack of resources were some of the barriers. ${ }^{14}$ The study done among 423 nurses to assess the practice of surgical site dressing shows $50 \%$ were practicing incorrectly. ${ }^{15}$

The study was limited to only one setting and non probability purposive sampling method was used which limits the scope of generalization of findings of the study.

\section{Conclusion}

Evidence-Based Practice guidelines should be established, and healthcare professionals should adhere to the infection control measures to reduce the incidence of SSI.

\section{Acknowledgments}

This study was supported by Dr. T.M.A Pai Endowment Chair on Antimicrobial Stewardship, Manipal Academy of Higher Education, Manipal.

\section{Appendix A. Supplementary data}

Supplementary data to this article can be found online at https:// doi.org/10.1016/j.cegh.2019.08.015.

\section{Conflicts of interest}

All authors report no conflict of interest relevant to this article.

\section{References}

1. World Health Organization. WHO guidelines on hand hygiene in health care. http:// whqlibdoc.who.int/publications/2009/9789241597906_eng.pdf; 2009.

2. Alp E, Elmali F, Ersoy S, Kucuk C, Doganay M. Incidence and risk factors of surgical site infection in general surgery in a developing country. Surg Today. 2014 Apr 1;44(4):685-689.

3. Shahane V, Bhawal S, Lele MU. Surgical site infections: a one year prospective study in a tertiary care center. Int J Health Sci. 2012 Jan;6(1):79.

4. Kumar A, Rai A. Prevalence of surgical site infection in general surgery in a tertiary care centre in India. International Surgery Journal. 2017 Aug 24;4(9):3101-3106.

5. Golia S, Nirmala AR. A study of superficial surgical site infections in a tertiary care hospital at Bangalore. Int J Res Med Sci. 2014;2(2):647-652.

6. Najm HY, Hussein RN. Assessment of wound dressing practices among nurses at the emergency hospitals in Erbil city. Zanco Journal of Medical Sciences (Zanco J Med Sci). 2018 Apr 1;22(1):96-103.

7. Maurya A, Mendhe S. Prevention of post-operative wound infection in accordance with evidence based practice. Int J Sci Res. 2014;3(7):1173-1177.

8. Wayne WD, Chad LC. Biostatistics A Foundation for Analysis in the Health Sciences. sixth ed. Canada: John Wiley; 1995.

9. Denise FP, Bernadette PH. Nursing Research Principles and Methods. sixth ed Philadelphia: Lippincott; 2004.

10. Nonino EA, Anselmi ML, Dalmas JC. Quality assessment of the wound dressing procedure in patients at a university hospital. Rev Latino-Am Enferm. 2008 Feb;16(1):57-63.

11. Wuerz T, Hanley M, Shaw R, Close R, Dow G. The impact of a standardized protocol on the quality of wound dressing procedures in hospitalized patients. Can J Infect Control. 2011 Sep 1;26(3).

12. Atiyah HH, Khudhur KM, Hasan SA. Evaluation of nurses' practices toward postoperative wound dressing in surgical wards. Nursing national Iraqi specility. 2012;25(1):29-39.

13. Gillespie B, Chaboyer W, Nieuwenhoven P, Rickard C. Drivers and barriers of surgical wound management in a large health care organisation: results of an environmental scan. Wound Practice \& Research: Journal of the Australian Wound Management Association. 2012 Jun;20(2):90.

14. Flanagan M. Barriers to the implementation of best practice in wound care. Wounds $U$ K. 2005;1(3):74

15. Teshager FA, Engeda EH, Worku WZ. Knowledge, practice, and associated factors towards prevention of surgical site infection among nurses working in Amhara regional state referral hospitals, Northwest Ethiopia. Surgery research and practice. $2015 ; 2015$. 